\title{
When, why and how foot orthoses (FOs) should be prescribed for children with flexible pes planus: a Delphi survey of podiatrists
}

\author{
Sindhrani Dars ${ }^{\text {Corresp., }}{ }^{1}$, Hayley Uden ${ }^{1}$, Saravana Kumar ${ }^{1}$, Helen A Banwell ${ }^{1}$ \\ ${ }^{1}$ School of Health Sciences, Sansom Institute for Health Research, University of South Australia, Australia \\ Corresponding Author: Sindhrani Dars \\ Email address: darsy009@mymail.unisa.edu.au
}

Background: Flexible pes planus (flat feet) in children is a common reason parents and caregivers seek health professionals consult and a frequent reason podiatrists prescribe foot orthoses. Yet no universal agreement exists on the diagnosis of this condition, or when and how foot orthoses should be prescribed. The aim of this study was to garner consensus and agreement among podiatrists on the use of FOs for paediatric flexible pes planus.

Methods: A three round Delphi consensus survey was undertaken with 15 podiatry experts from Australia, New Zealand and the United Kingdom. Round one gathered consensus on the diagnosis and intervention into paediatric pes planus with specific questions on types of FOs and prescription variables used. Round two and three were based on answers from Round one and gathered agreement (rationale for choices) on a five point Likert scale. $70 \%$ of respondents had to agree to a statement for it to be accepted as consensus or agreement.

Results: Consensus and agreement was achieved for 83 statements directing the diagnosis of pes planus (using FPI-6 and/or rearfoot measures), common signs and symptoms (e.g. pain, fatigue, abnormal gait and other functional concerns) that direct when to intervene into paediatric flexible pes planus. Prefabricated orthoses were the preferred intervention where adequate control is gained with their use. When customised orthoses are prescribed, a vertical [heel] cast pour (71.4\%) and minimal arch fill (76.9\%) are the prescription variables of choice, plus or minus additional variables (i.e. medial heel (Kirby) skive, the use of a University of California Biomechanical Laboratory device or a medial flange) dependent on level of disorder and plane of excessive motion.

Conclusions: This study identified consensus and agreement on a series of diagnosis methods and interventions for the paediatric flexible pes planus. A clinical protocol was developed from the resultant consensus statements which provides clinicians with a series of evidenced-informed statements to better guide them on the when, how and why FOs are used specific to this population. 
"When, Why and How Foot 2

Orthoses (FOs) should be

prescribed for children with

" flexible pes planus: A Delphi

s survey of Podiatrists"

6

7 Sindhrani Dars ${ }^{1}$, Hayley Uden ${ }^{1}$, Dr. Saravana Kumar ${ }^{1}$ and Dr. Helen A Banwell ${ }^{1}$.

8

$9{ }^{1}$ School of Health Sciences, Sansom Institute for Health Research, University of South Australia.

11 Corresponding Author:

12 Sindhrani Dars ${ }^{1}$

13 Email address: darsy009@mymail.unisa.edu.au 
15 Abstract

16 Background: Flexible pes planus (flat feet) in children is a common reason parents and

17 caregivers seek health professionals consult and a frequent reason podiatrists prescribe foot orthoses. Yet no universal agreement exists on the diagnosis of this condition, or when and how foot orthoses should be prescribed. The aim of this study was to garner consensus and agreement among podiatrists on the use of FOs for paediatric flexible pes planus.

Methods: A three round Delphi consensus survey was undertaken with 15 podiatry experts from Australia, New Zealand and the United Kingdom. Round one gathered consensus on the diagnosis and intervention into paediatric pes planus with specific questions on types of FOs and prescription variables used. Round two and three were based on answers from Round one and gathered agreement (rationale for choices) on a five point Likert scale. $70 \%$ of respondents had to agree to a statement for it to be accepted as consensus or agreement.

Results: Consensus and agreement was achieved for 83 statements directing the diagnosis of pes planus (using FPI-6 and/or rearfoot measures), common signs and symptoms (e.g. pain, fatigue, abnormal gait and other functional concerns) that direct when to intervene into paediatric flexible pes planus. Prefabricated orthoses were the preferred intervention where adequate control is gained with their use. When customised orthoses are prescribed, a vertical [heel] cast pour (71.4\%) and minimal arch fill (76.9\%) are the prescription variables of choice, plus or minus additional variables (i.e. medial heel (Kirby) skive, the use of a University of California Biomechanical Laboratory device or a medial flange) dependent on level of disorder and plane of excessive motion. 
36 Conclusions: This study identified consensus and agreement on a series of diagnosis methods

37 and interventions for the paediatric flexible pes planus. A clinical protocol was developed from

38 the resultant consensus statements which provides clinicians with a series of evidenced-informed

39 statements to better guide them on the when, how and why FOs are used specific to this

40 population.

41

42 


\section{Background}

44 Flexible pes planus (flat feet) describes feet with a lowered medial longitudinal arch on weight

45 bearing, that resolves when non-weight bearing (Fabry 2010; Harris et al. 2004; Roth et al.

46

47

48

49

50

51

52

53

54

55

56

57

58

2013). It is distinct from rigid pes planus, a congenital, rigid or spastic deformity of the foot, affecting less than $1 \%$ of the population and often requiring surgical management (Harris et al. 2004; Rome et al. 2010; WHO 2016). Prevalence of paediatric pes planus ranges from $48.5 \%$ to 77.9\% in children aged 2-16 years (Chen et al. 2014; Evans \& Rome 2011; Halabchi et al. 2013), reducing to only $2-23 \%$ in the adult population (Dunn et al. 2004; Golightly et al. 2012;

Kosashvili et al. 2008). The larger prevalence observed in children can be explained by many factors, including age appropriate ligament laxity, and advancing maturation of neuromuscular control (Lin et al. 2001; Pfeiffer et al. 2006; Stavlas et al. 2005; Uden et al. 2017), with most of the arch 'flatness' reducing over the first decade of life (El et al. 2006; Rome et al. 2010; Stavlas et al. 2005). However, despite being an expected finding in typically developing children (Uden et al. 2017) flexible paediatric pes planus is one of the most frequently cited orthopaedic concerns that prompt parents and caregivers to seek health practitioner advice (Fabry 2010; Rome et al. 2010). Whilst parental concern may stem from aesthetics, there is evidence that some children with flexible pes planus present with symptoms associated with their foot posture (i.e. symptomatic flexible pes planus). Children with symptomatic flexible pes planus have been shown to have reduced walking velocity $(\mathrm{p}<0.05)$ (Kothari et al. 2015; Lin et al. 2001), poorer performance in lower-limb involved physical tasks (Lin et al. 2001), and are significantly more likely to have hip, knee (odds ratio $=1.33, \mathrm{p}<0.01)$ and back pain (odds ratio $=1.22, \mathrm{p}=0.01)$ (Kothari et al. 2016). Quality of life scores assessed by the Oxford Ankle Foot Questionnaire for Children (OxAFQ_C) highlight that children with symptomatic flexible pes planus have 
66 significantly lower scores when compared to children with 'normal' arches, particularly in the

67 physical domain (66.7\% vs 91.7\%, p<0.05) (Kothari et al. 2015). Furthermore, flexible pes

68 planus that persists or presents in adulthood is associated with increased rates of intermittent

69 lower back pain, anterior knee pain, joint degeneration, instability and functional limitations

70 and/or disability (Dunn et al. 2004; Golightly et al. 2012; Kosashvili et al. 2008). The challenge

71 for health professionals is to determine when intervention for paediatric flexible pes planus may

72 warranted to ameliorate symptoms and reduce complications later in life (Dunn et al. 2004;

73 Golightly et al. 2012; Kosashvili et al. 2008).

74 The most frequently cited intervention for paediatric pes planus is foot orthoses (FOs) (Halabchi

75 et al. 2013; MacKenzie et al. 2012; Rome et al. 2010; Wenger et al. 1989; Whitford \& Esterman

76 2007). Other common non-surgical treatments include footwear, activity modification, weight

77 reduction, manipulations, serial castings, and stretch exercises of gastrocnemius-soleal complex

78 (Halabchi et al. 2013; Harris et al. 2004; Rome et al. 2010), yet little is understood on how or

79 when different interventions are used.

80 Minimal guidance exists for clinicians on diagnosis and intervention pathways specific to

81 symptomatic presentations of paediatric flexible pes planus. The paediatric flatfoot proforma (p-

82 FFP), developed by Evans (2008), offers direction when management may be required (Evans

83 2008), however, there is no reference standard on what symptoms of flexible pes planus should

84 be cause for intervention, or indeed, no consensus on what intervention methods should be used.

85 Without appropriate tools to guide practice, clinicians are guided by their clinical experience and

86 judgement only, potentially resulting in disparity amongst professionals and outcomes of

87 management alike. This identifies the need for developing a clinical protocol that not only 
88 identifies the commonly encountered signs and symptoms of paediatric flexible pes planus but

89 also helps to identify the treatment options being utilised in the clinical practice.

90 In the absence of appropriate evidence-informed tools, , consensus surveys such as the Delphi

91 method, are a reliable and valid method of determining expert opinion (Van der Linde et al.

92 2005; Vernon 2009). This study primarily aimed to garner consensus and agreement, with Delphi

93 consensus survey, from experienced podiatrists of Australia (AU), New Zealand (NZ) and

94 United Kingdom (UK), on the presentation and management of paediatric flexible pes planus.

95 The secondary aim was to develop a clinical protocol based on this expert opinion to direct

96 clinicians on when, why and how FOs should be prescribed for children with symptomatic

97 flexible pes planus

98 Methods

99 Study design

100 The study was a three-round modified Delphi panel design where participants' opinion was

101 sought in Round one, with responses collated and analysed for consensus (Hasson et al. 2000;

102 Hsu \& Sandford 2007; Vernon 2009). Responses not reaching consensus were reviewed by

103 participants in successive rounds for consideration, commenting and ranking of agreement

104 (Figure 1). The study was approved by the University of South Australia's Human Research

105 Ethics Committee (Protocol no. 0000035501). All participants provided written informed

106 consent. 
107 Participants

108 Fifteen podiatrists were recruited for the Delphi survey panel. Potential participants were

109 required to be a registered and practicing podiatrist with clinical experience of $\geq 10$ years, or

110 have worked in a paediatric-focused position for $\geq 8$ years. The participants' inclusion was also

111 based on satisfying at least one of the following criteria: held an academic position teaching

112 paediatric podiatry/clinical practice within a podiatry program; held a clinical position with

113 practice focused on paediatric assessment and intervention; or had published research on

114 paediatric theory/FOs within past five years.

115 Participants were recruited from Australia, New Zealand and the United Kingdom. These three

116 countries were chosen due to similarities in podiatry undergraduate education, scope of podiatric

117 practice and health care contexts. A total of 38 potential participants were identified based on the

118 above criteria, international reputation, employment within 'paediatric' specific public health

119 roles, involvement in published research on paediatric podiatry/FOs, and staff listings of

120 academic institutes. Participants were then randomly selected from this list of potential

121 participants and invited to participate until 15 experts were enrolled. This number of participants

122 offers a broad sample of podiatry expertise whilst remaining pragmatic as a manageable panel

123 size (Hasson et al. 2000; Hsu \& Sandford 2007; Van der Linde et al. 2005; Vernon 2009).

\section{Procedure}

125 Potential participants were invited to participate (Data S1). A preliminary survey was completed

126 to ensure eligibility for the study and informed consent to participate was obtained electronically.

127 Once enrolled, participants were given four weeks to complete each round. Late responders were

128 sent a reminder with further two weeks' extension. Participants were considered non-responders 
129 if they failed to complete the survey within six weeks of the distribution date and had not

130 requested extra time. The participants were reminded in each round that the focus of this study

131 was on flexible pes planus in otherwise typically developing children i.e. not associated with

132 neurological, muscular or structural disease or abnormalities.

\section{Survey Format}

134 A Delphi survey consisting of three rounds was conducted (Figure 1). Data was collected using 135 online survey platform Survey Monkey® (SurveyMonkey Inc, Palo Alto, California, USA).

136 Round one was developed based on common assessment questions including child's subjective

137 history, as described in the GALLOP assessment tool developed (Cranage et al. 2016) and 138 common prescription variables for foot orthoses as established from previous work in an adult 139 population (Banwell et al. 2014). Prior to conducting the survey, the preliminary survey and

140 Round one was piloted by two Australian podiatrists independent $t$ study, for feedback on 141 structure, clarity, ordering and framing of the questions. These podiatrists were excluded from 142 the main study. All three Delphi rounds were divided into four sections: Establishing the 143 presence of flexible pes planus; intervention into flexible pes planus; using FOs for flexible pes 144 planus in children; and approach to prescription of FOs for flexible pes planus in children (Data 145 S2). Section one and two included questions on the method of clinical diagnosis and signs and 146 symptoms associated with paediatric flexible pes planus that may initiate intervention. Section 147 three and four included questions on participants' preference for the FOs prescribed for this 148 population, including when and how customised or pre-fabricated orthoses would be used and when alternative interventions may be considered (e.g. footwear modification and rearfoot

150 wedges). Specific questions on their use of different prescription variables were also included, 151 with participants asked to describe their thoughts on desired outcomes of FOs intervention for 
152 paediatric flexible pes planus. Participants were supplied a glossary of terms to accommodate

153 variations in terminology between countries (Data S3).

154

155

Figure 1: Flow diagram of Delphi process with number of statements in each round

156 Round one included a total of 31 questions requiring participants to supply closed and open-

157 ended responses. The closed-ended questions were directly analysed for consensus by two

158 authors (SD, HB). The open-ended responses were themed into statements and analysed for

159 consensus by the same two authors (SD, HB), with recommendations from the third author (HU)

160 if disagreement arose. Statements were considered to have reached consensus within Round one

161 when $70 \%$ or more of participants indicated the same statement. Statements were required to

162 receive $70 \%$ consensus to be accepted, thus remaining consistent with existing literature

163 (Banwell et al. 2014; Bisson et al. 2010; Cranage et al. 2016; Mokkink et al. 2010; Vernon et al.

164 2003). Round two was based on the statements developed from Round one (Figure 1).

165 Participants were requested to consider each question in relationship to the presentation of a

166 child between the ages 0-18 with flexible pes planus and indicate their level of agreement on a

167 five point Likert scale (i.e. strongly disagree, disagree, neutral, agree or strongly agree) (Likert

168 1932), and comment further if desired. As an example, statements related to the likelihood of

169 prescribing FOs in the presence of pain are displayed in Figure 2.

172 In Round two and three, statements were considered accepted if $70 \%$ or more of participants

173 indicated that they agreed or strongly agreed with a statement. Statements not reaching $50 \%$ 
$17550-69 \%$ agreement were reviewed in subsequent survey rounds, when available, to ensure

176 adequate panel consideration. Statements were also excluded if agreement had not been achieved

177 within two rounds.

178 The a priori decision was that the Delphi would be concluded when the response rate dropped

179 below $70 \%$ or when Round three was completed, irrespective of agreement. Participants were

180 asked to keep their involvement confidential and participants were asked to maintain intra-panel

181 communication anonymity throughout the survey.

\section{Data management and analysis}

183 The outcomes of interest were consensus and agreement. Consensus was sought in Round one

184 only (Figure 1). Consensus was achieved with $\geq 70 \%$ participants ( $\geq 11$ of 15 participants)

185 indicated a consistent response for the open and closed ended questions (Data S2). Agreement

186 was sought in Rounds two and three (Figure 1). Agreement was achieved when $\geq 70 \%$ ( $\geq 10$ of

18714 participants) agreed or strongly agreed to a given statement based on a five-point Likert scale

188 (Figure 1).

189 The further comments received in Round one and two were collated and themed into statements

190 by two authors (SD and HB) to be reviewed in subsequent rounds. Participants were provided

191 with tabled presentations of the data from previous rounds, an example of this can be seen in

192 Figure 3.

Figure 3: An example question from Round three

195 Data not relating to the scope of the study, i.e. flexible pes planus in otherwise normally

196 developing children and conservative management, were excluded. Statements not reaching 
197 consensus or agreement by the end of Round three were also excluded (Data S4). Descriptive

198 data analysis was undertaken in Microsoft Excel 2016 (Microsoft Corporation, Redmond

199 Washington, USA). 


\section{Participant's characteristics}

202 Nine of the participants were Australian (64.3\%), three were from the United Kingdom (21.5\%)

203 and two from New Zealand (14.5\%). Overall, 57\% of participants were male (8:6 males:females

204 ratio) with an average age of 39.1 years (SD 4.8, range $=33-46$ years). Participants had been

205 practicing for an average of 16.9 years (SD 5.6, range 8-28 years) and $78.6 \%$ either held or were

206 working towards a recognised post-graduate qualification. Over 70\% of the panel (71.4\%) listed

207 more than one employment setting with clinicians $(n=11)$ and academics $(n=9)$ reaching almost

208 equal representation. Four participants also identified themselves as researchers. Only two

209 participants identified themselves as academics only (Table 1).

\section{Table 1: Participants' characteristics}

\section{Survey Findings}

\section{Consensus}

213 Round one received $100 \%$ response rate $(15 / 15)$ and resulted in 21 statements reaching consensus and 173 statements to be reviewed in subsequent rounds (Table 2). Consensus was

215 reached for the diagnosis of paediatric flexible pes planus to be determined using an assessment 216 tool such as the Foot Posture Index - six item version (FPI-6) or the Paediatric Flat Foot

217 Performa (pFFF) (80.0\% consensus), or by visually assessing static foot posture $(73.3 \%$

218 consensus) including resting and neutral calcaneal position (84.6\% consensus). For

219 determination of foot function in paediatric flexible pes planus population, three techniques

220 reached consensus: range of motion (100\% consensus); visual gait analysis (93.3\% consensus); 
221 and muscle strength (93.3\% consensus), (Table 2$)$. The signs and symptoms that were considered

222 to increase the likeliness of FOs prescription were foot pain (93.3\% consensus), lower leg pain

223 (73.3\%), activity limitation ( $73.3 \%$ consensus) and a severe abnormal foot posture, such as two

224 standard deviations from the expected measure (78.6\% consensus). Conversely, the panel

225 collectively agreed that that the age or weight of the child does not influence the decision to use

226 FOs (71.4\% agreement and 92.3\% consensus respectively), (Table 2).

227 For management of paediatric flexible pes planus, the use of pre-fabricated FOs was preferred

228 over customised FOs $(74.0 \pm 7.1 \%$, range $5-100$ vs $34.0 \pm 9.3 \%$, range $0-100)$, (Table 2$)$. In the

229 event that customised FOs are prescribed, the specific prescription variables that should be used

230 are; a neutral or vertical (heel) cast pour (71.4\% consensus), and a minimal arch fill (76.9\%

231 consensus), (Table 2). Whereas eight prescription variables received negative consensus, that is,

232 they should not be used for customised FOs prescribed for paediatric flexible pes planus (Table

233 2). These variables were: maximum arch fill (72.7\% consensus); everted cast pour (91.6\%

234 consensus); everted rearfoot post (90.0\% consensus); Blake inverted device $\left[>15^{\circ}\right](84.6 \%$

235 consensus); Blake inverted rearfoot post $\left[>15^{\circ}\right](90.0 \%$ consensus $)$; rearfoot post with motion

236 (88.9\% consensus); and inverted or everted forefoot post (70.0\% and $77.8 \%$ consensus

237 respectively). No further consensus was achieved.

238

Table 2: Statements reaching consensus $(\geq 70 \%)$ in Round One

Agreement

240 Round two and three both received 93.3\% response rate (14/15), (Figure 1). Of the 152

241 statements reviewed in Round two, 44 achieved agreement (Table 3), 36 received between 50-

$24269 \%$ agreement, 72 were excluded (Data S4) and seven new statements were generated from 
243 comments received (Figure 1). Of the 43 statements reviewed in Round three, 18 statements

244 achieved agreement (Table 3), all other statements were excluded, (Figure 1). No further

245 comments were sought in Round three (Figure 1).

246 Of the 62 accepted statements, eight reached 100\% agreement (Table 3). These were: use of Foot

247 Posture Index-6 (FPI-6) to determine the presence of flexible pes planus; conducting balance

248 tests to determine the functional impact of pes planus; and to prescribe FOs in presence of

249 symptoms correlating to WHO-ICD (World Health Organisation International Classification of

250 Diseases), gross pronation due to an apropulsive gait and low tone, and also other symptoms

251 such as general discomfort, foot and leg pain, reduced walking ability, poor endurance and

252 balance, affected function and gross motor skills, pain of the Tibialis Posterior tendon and

253 Medial Tibial Stress Syndrome (MTSS) type symptoms (Table 3).

254 There was high agreement that flexible pes planus may be assessed by visual assessment of foot

255 in gait with dynamic weight bearing and non-weight bearing foot measures (85.7\% agreement)

256 (Table 3). The techniques and balance tests to assess foot function in pes planus included:

257 neurological assessment (78.5\% agreement); single limb balance (71.4\% agreement); hopping

258 (78.5\% agreement); running (78.5\% agreement); jumping (71.4\% agreement); walking along a

259 straight line with marching and heel-to-toe gait (78.5\% agreement); and timed balance test with

260 eyes opened and closed (85.7\% agreement).

261 Other pain or symptoms that increase the likeliness of intervention included: activity related pain

262 (92.8\% agreement); affected dynamic foot function (85.7\% agreement); presence of structural

263 changes (71.4\% agreement); delayed milestones (78.5\% agreement); hereditary lower limb

264 conditions causing pain or changing function (92.8\% agreement); and pain in plantar fascia or 
265 heel (92.8\% and 78.5\% agreement respectively). Whereas parental concern would only result in

266 prescription of FOs if accompanied by affected function (78.5\% agreement), (Table 3).

267 The panel also agreed on prescribing FOs for paediatric pes planus based on the extent of

268 pathology, degree of deformity, acquisition of motor skills, activity levels and function rather

269 than a specific age of the child (92.8\% agreement) (Table 3$)$. The aim of prescribing FOs, that is

270 expected outcomes of treatment that were agreed upon, included: reduction of symptoms and

271 fatigue (92.8\% and $85.7 \%$ agreement respectively); improving gross motor skills (85.7\%

272 agreement); improving balance, stability, comfort, coordination, stamina and endurance $(92.8 \%$

273 agreement); and overall wellbeing and health outcomes which will in turn increase the overall

274 quality of life for children with flexible pes planus ( $71.4 \%$ agreement). The rationale behind

275 preferring pre-fabricated orthosis over customised were pre-fabricated FOs being easily

276 modifiable (78.5\% agreement), cost effective (71.4\% agreement) and require less time to

277 dispense (78.5\% agreement). Other desirable features of pre-fabricated FOs identified include

278 good fitting in shoes (71.4\% agreement), smooth contour thus increased comfort $(71.4 \%$

279 agreement), size availability (78.5\% agreement), and appropriate strength of material to provide

280 adequate control (85.7\% agreement). Furthermore, participants were unanimous in agreeing

$281(100 \%)$ that customised FOs should be used when prefabricated devices do not supply adequate

282 support for the child's foot (Table 3).

283 When customised FOs are to be used, a medial (Kirby) heel skive can be prescribed when severe 284 pes planus exists in frontal plane (71.4\% agreement), to provide better rearfoot control $(78.5 \%$ 285 agreement), or to reduce subtalar joint pronation (85.7\% agreement) (Table 3). Agreement was 286 reached that a UCBL device (which has both medial and lateral flange in-situ) can be used in the 287 presence of grossly pronated feet with hypertonia (71.4\% agreement), and to provide midfoot 
288 control for transverse plane motion (92.8\% agreement). A medial flange alone could be

289 prescribed to prevent the foot rolling over the device or if the aim of the device is to limit

290 midtarsal joint pronation $(78.5 \%$ agreement) or when extra midfoot control is required $(92.8 \%$

291 agreement) or if the child cannot tolerate the medial edge of the device due to having very

292 flexible pes planus (71.4\% agreement). It was also agreed to accommodate talo-navicular bulge

293 by including a wider midfoot area in the customised FOs (71.4\% agreement). Other alternative

294 devices used to treat flexible pes planus as agreed upon, include rearfoot or heel wedges $(71.4 \%$

295 agreement) and exercise therapy (85.7\% agreement). Over 70\% of the panel also agreed on using

296 three-dimensional printing shell materials for customised FOs when available $(71.4 \%$

297 agreement), (Table 3)

298

299

300

301

302

303

304

305

306

307

308

309

\section{Table 3: Statements receiving agreement of $\geq 70 \%$ from Round two and three of} Delphi

Abbreviations: WB: Wight bearing; WHO-ICD: World Health Organisation-International Classification of Diseases; STJ: Subtalar joint; MTJ: Midtarsal joint; and UCBL: University of California Biomechanics Laboratory.

At the completion of the three rounds, 83 statements (21 consensus and 62 agreement) were accepted on when, why and how orthoses are prescribed for paediatric flexible pes planus (Figure 1, Table 2 and 3).

\section{Development of consensus-based clinical protocol}

Following analysis of the Delphi survey findings, all included consensus and agreement statements were compiled to construct 'A clinical protocol for paediatric flexible pes planus' 
310 (Figure 4). The protocol consists of three main sections: Confirm diagnosis; Signs and

311 symptoms; and Intervention.

312 The protocol firstly allows clinicians to record the method of diagnosis (Figure 4). Secondly, 313 using a simple 'tick and flick' flow chart, the observed and reported signs and symptoms can be

314 indicated. The symptom of pain is sub-divided into different regions, based on the agreement of

315 the expert panel, with functional symptoms such as reported fatigue and perceived excessive

316 tripping also included (Figure 4). A section for identifying different signs specific to flexible pes

317 planus and its history was also included for clinicians to tick appropriate options. These

318 included: gross pronation; gait abnormalities; reduced range of motion (ROM); reduced muscle

319 strength; activity limitation; affected function during single leg stance, walking, running and

320 turning; poor endurance and balance; diagnosed Developmental Coordination Disorder (DCD);

321 structural changes; delayed milestones and gross motor skill acquisition; neurological concerns

322 including absent reflexes, affected sensation and low tone; and hereditary limb disorders

323 changing foot function.

324 Finally, the protocol directs clinicians to consider prefabricated FOs in the first instance,

325 confirming adequate fit and control is achieved, with recommendations for the prescription of

326 customised FOs if this is not achieved (Figure 4). Other alternative or additional conservative

327 interventions or treatments are listed to direct the clinician to consider: footwear modification;

328 activity modifications; strengthening exercises and stretching exercises where required.

329 This clinical protocol can be used concurrently with alternative tools such as the pFFP by Evans

330 (2008) and Harris et al. 2004 flow chart, to help clinicians follow a logical pattern for

331 management of paediatric flexible pes planus. 


\section{Discussion}

335

336

337

338

339

340

341

342

343

344

345

346

347

348

349

350

351

352

353

354

Despite its common presentation in clinical practice, to date, there has been limited research

which has systematically investigated how best to assess, diagnose and treat paediatric flexible

pes planus. This has resulted in persistent knowledge gaps in this area. This research aims to address this knowledge gap by gathering expert international podiatry opinion on the presentation, diagnoses and intervention for paediatric flexible pes planus. The panel concluded that age and weight are not influencing factors for intervention, however, the presence of symptoms, degree of deformity or determinants of function were influential. When management is required, pre-fabricated orthoses are preferred by clinicians if they provide adequate support. Customised FOs, with targeted prescription options, should be prescribed when support is not adequately achieved with pre-fabricated devices.

While the literature acknowledges paediatric flexible pes planus as a frequently observed concern, there is considerable debate on its diagnosis (Pfeiffer et al. 2006; Roth et al. 2013;

Tudor et al. 2009), and whether or not intervention is required (Pfeiffer et al. 2006; Tudor et al. 2009; Whitford \& Esterman 2007). A 2010 Cochrane systematic review on the efficacy of nonsurgical intervention for paediatric flexible pes planus (Rome et al. 2010) concluded that very limited high level evidence exists on this topic with equivocal findings for intervention, specifically FOs. However, this review was based on only three randomised control trials (Powell et al. 2005; Wenger et al. 1989; Whitford \& Esterman 2007), with only one of the studies using a symptomatic population. Indeed, much of the research on the use of FOs for flexible pes planus in children, to date, has been focussed on non-symptomatic populations 
355 (Aboutorabi et al. 2014; Bleck \& Berzins 1977; Bok et al. 2014; Bordelon \& Lusskin 1980;

356 Capasso 1993; Riccio et al. 2009; Valmassy \& Terrafranca 1986). The findings of this research

357 demonstrate that it is the presenting symptoms of a child with flexible pes planus that guide the

358 podiatrists' decision to intervene along with clinical signs of the condition (observations and

359 measurements). Furthermore, this research has determined that pain is only one of the symptoms

360 that should initiate treatment, with presentations of: activity limitation; fatigue; perceived

361 excessive tripping; and decline in function, strength and endurance also contributing to

362 'symptomatic' flexible pes planus. These findings are in line with previous literature (Evans

363 2008; Halabchi et al. 2013; Harris et al. 2004; Whitford \& Esterman 2007), which found that

364 children with flat feet, when compared to children with typically developing arches, have lower

365 physical performance in tasks including squatting and standing, standing on toes, toe walking,

366 heel walking and one leg standing and hoping ( $\mathrm{p}<0.02)$ (Lin et al. 2001) and experience

367 increased knee (odds ratio $=1.33, \mathrm{p}<0.01)$, hip and back pain (odds ratio $=1.22, \mathrm{p}=0.01)$

368 (Kothari et al. 2016); which negatively affects their quality of life (Kothari et al. 2015). As such

369 the aim of intervention, as agreed by panellists, was to reduce these frequently observed

370 symptoms which potentially increases the overall quality of life for children with symptomatic

371 flexible pes planus.

372 It was determined that interventions for pes planus was inclusive of FOs, footwear changes,

373 activity modifications and stretching and strengthening exercises. Within the literature and

374 anecdotally, FOs are often cited as a frontline management strategy for pes planus (Halabchi et

375 al. 2013; MacKenzie et al. 2012; Rome et al. 2010; Wenger et al. 1989; Whitford \& Esterman

376 2007). There is a lack of universally accepted pathway that defines when and how foot orthoses

377 should be used in the management of paediatric flexible flatfeet (Stavlas et al. 2005; Tudor et al. 
378 2009). Assessment tools, such as the p-FFP (Evans 2008) and Harris et al. clinical pathway

379 (2004), assist in guiding the practitioner, however the p-FFP does not identify the specific

380 intervention modalities and Harris et al. (2004) includes rigid pes planus and a surgical focus to

381 their interventions. This protocol adds to the existing literature by detailing the specific signs and

382 symptoms that are presented in the clinical practice and detailing specifics on intervention. For

383 example, this research determined that pre-fabricated orthoses are preferred by participants due

384 to cost effectiveness, timeliness of dispense and ease of modification. Customised FOs, however,

385 were recommended when adequate support was not gained with the use of prefabricated devices,

386 and with targeted prescription dependant on the 'level of control' required. Despite this

387 preferential reasoning, it is importance to acknowledge that limited evidence exists for the use of

388 one type of FOs over another (MacKenzie et al. 2012; Rome et al. 2010), with no known studies

389 comparing the impact of different prescription variables within a paediatric population. The

390 results of this research may assist in developing future research which have a particular focus on

391 informing clinical decision making. Specifically, on which prescription variables are frequently

392 used by clinicians for symptomatic pes planus. Moreover, selecting specific prescription

393 variables depending on different features of the deformity. As a starting point for translating best

394 available evidence into clinical practice, this research has developed a clinical protocol which

395 can offer clinicians, researchers and other stakeholders' in this field opportunities for evidence-

396 informed assessment, diagnosis and intervention for paediatric flexible pes planus.

397 There are some limitations to this Delphi survey. Firstly, the absence of agreement on a

398 universally accepted definition of paediatric flexible pes planus. It was clearly established at the

399 commencement of the study that the aim was a non-pathological flexible pes planus in children

400 that was not associated with any muscular, neurological or osseous abnormalities. Secondly, 
401 even though this research provides evidence of consistency in podiatric practice for paediatric

402 flexible pes planus, being a Delphi survey methodology, it is considered as an expert opinion

403 only. As the participants were selected from three different countries with extensive clinical

404 expertise, this research sought to capture broad and diverse opinions. Moreover, participants

405 were not required to declare any potential conflicts of interest (including vested interests in foot

406 orthoses manufacture/brand ownership), however, the research questions were framed around

407 specific features of FOs only, excluding specific 'brands'. This is specifically prudent given two

408 sets of participants reported sharing a common place of employment. As part of the research

409 process, participants were reminded of the importance of academic rigor in terms of remaining

410 anonymous, strategies were put in place to minimise collusion and the definition of "expert" was

411 based on current podiatry literature (Banwell et al. 2014; Okoli \& Pawlowski 2004; Vernon

412 2009). Furthermore, due to limited psychometric testing of individual diagnostic measures used

413 for paediatric pes planus (Evans et al. 2009), the measures indicated in this research should be

414 used with caution and with clinical experience and judgement.

\section{Conclusion}

416 Flexible paediatric pes planus presents a dichotomy in clinical practice. While it is commonly

417 encountered, to date there has been minimal guidance on how best to assess, diagnose and treat

418 it. This research, by bringing together experienced podiatrists in this field from across three

419 different counties, has generated expert-informed statements which can be used to guide clinical

420 practice. In order to facilitate timely and effective translation to clinical practice and promote

421 evidence-based practice, a ready-to-use clinical protocol provides clinicians with the opportunity

422 to complement their clinical expertise with current best available evidence while sharing the

423 decision making with parents and caregivers. 


\section{Abbreviations}

426 FOs: Foot Orthoses; OxAFQ_C: Oxford Ankle Foot Questionnaire for Children; pFFP: The paediatric

427 flatfoot proforma; AU: Australia; NZ: New Zealand; UK: United Kingdom; FPI: Foot Posture Index;

428 RCSP: Resting Calcaneal Stance Position; NCSP: Neutral Calcaneal Stance Position; WB: Weight

429 Bearing; WHO-ICD: World Health Organisation - International Classification of Diseases; MTSS:

430 Medial Tibial Stress Syndrome; STJ: Subtalar joint; MTJ: Midtarsal joint; UCBL: University of

431 California Biomechanics Laboratory; RCTs: Randomised Controlled Trials.

432 Acknowledgements: The Authors would like to thank the podiatrists involved with piloting the surveys 433 and sincerely acknowledge the contribution of the expert panel towards this research. 
434

435

436

437

438

439

440

441

442

443

444

445

446

447

448

449

450

451

452

453

454

455

456

457

458

459

460

461

462

463

464

465

466

467

468

469

470

471

472

473

474

475

476

477

478

479

480

\section{References}

Aboutorabi A, Saeedi H, Kamali M, Farahmand B, Eshraghi A, and Dolagh RS. 2014. Immediate effect of orthopedic shoe and functional foot orthosis on center of pressure displacement and gait parameters in juvenile flexible flat foot. Prosthetics and Orthotics International 38:218-223.

Banwell HA, Mackintosh S, Thewlis D, and Landorf KB. 2014. Consensus-based recommendations of Australian podiatrists for the prescription of foot orthoses for symptomatic flexible pes planus in adults. Journal of Foot and Ankle Research 7:1-13. 10.1186/s13047-014-0049-2

Bisson JI, Tavakoly B, Witteveen AB, Ajdukovic D, Jehel L, Johansen VJ, Nordanger D, Garcia FO, Punamaki R-L, Schnyder U, Sezgin AU, Wittmann L, and Olff M. 2010. TENTS guidelines: development of post-disaster psychosocial care guidelines through a Delphi process. The British Journal of Psychiatry 196:69-74. 10.1192/bjp.bp.109.066266

Bleck EE, and Berzins UJ. 1977. Conservative management of pes valgus with plantar flexed talus, flexible. Clinical Orthopaedics and Related Research 122:85-94.

Bok SK, Kim BO, Lim JH, and Ahn SY. 2014. Effects of custom-made rigid foot orthosis on pes planus in children over 6 years old. Annals of Rehabilitation Medicine 38:369-375. 10.5535/arm.2014.38.3.369

Bordelon RL, and Lusskin R. 1980. Correction of Hypermobile Flatfoot in Children by Molded Insert. Foot \& Ankle 1:143-150. 10.1177/107110078000100303

Capasso G. 1993. Dynamic varus heel cup: a new orthosis for treating pes planovalgus. Italian journal of orthopaedics and traumatology 19:113-123.

Chen K-C, Tung L-C, Tung C-H, Yeh C-J, Yang J-F, and Wang C-H. 2014. An investigation of the factors affecting flatfoot in children with delayed motor development. Research in developmental disabilities 35:639-645.

Cranage S, Banwell H, and Williams CM. 2016. Gait and Lower Limb Observation of Paediatrics (GALLOP): development of a consensus based paediatric podiatry and physiotherapy standardised recording proforma. Journal of foot and ankle research 9:1-10.

Dunn J, Link C, Felson D, Crincoli M, Keysor J, and McKinlay J. 2004. Prevalence of foot and ankle conditions in a multiethnic community sample of older adults. American journal of epidemiology 159:491-498.

El O, Akcali O, Kosay C, Kaner B, Arslan Y, Sagol E, Soylev S, Iyidogan D, Cinar N, and Peker O. 2006. Flexible flatfoot and related factors in primary school children: a report of a screening study. Rheumatology International 26:1050-1053. 10.1007/s00296-006-0128-1

Evans AM. 2008. The flat-footed child-to treat or not to treat: what is the clinician to do? Journal of the American Podiatric Medical Association 98:386-393.

Evans AM, Nicholson H, and Zakarias N. 2009. The paediatric flat foot proforma ( $p$-FFP): improved and abridged following a reproducibility study. Journal of foot and ankle research 2.

Evans AM, and Rome K. 2011. A Cochrane review of the evidence for non-surgical interventions for flexible pediatric flat feet. European journal of physical \& rehabilitation medicine 47:69-89.

Fabry G. 2010. Clinical practice: Static, axial, and rotational deformities of the lower extremities in children. European Journal of Pediatrics 169:529-534.

Golightly YM, Hannan MT, Dufour AB, and Jordan JM. 2012. Racial differences in foot disorders and foot type. Arthritis Care \& Research 64:1756-1759. 10.1002/acr.21752

Halabchi F, Mazaheri R, Mirshahi M, and Abbasian L. 2013. Pediatric Flexible Flatfoot; Clinical Aspects and Algorithmic Approach. Iranian Journal of Pediatrics 23:247-260.

Harris EJ, Vanore JV, Thomas JL, Kravitz SR, Mendelson SA, Mendicino RW, Silvani SH, and Gassen SC. 2004. Diagnosis and treatment of pediatric flatfoot. The Journal of Foot and Ankle Surgery 43:341-373. 10.1053/j.jfas.2004.09.013 
481

482

483

484

485

486

487

488

489

490

491

492

493

494

495

496

497

498

499

500

501

502

503

504

505

506

507

508

509

510

511

512

513

514

515

516

517

518

519

520

521

522

523

524

525

526

527

528

Hasson F, Keeney S, and McKenna H. 2000. Research guidelines for the Delphi survey technique. Journal of Advanced Nursing 32:1008-1015.

Hsu C-C, and Sandford BA. 2007. The Delphi technique: making sense of consensus. Practical assessment, research \& evaluation 12:1-8.

Kosashvili Y, Fridman T, Backstein D, Safir O, and Ziv YB. 2008. The Correlation between Pes Planus and Anterior Knee or Intermittent Low Back Pain. Foot \& ankle international 29:910-913. 10.3113/FAI.2008.0910

Kothari A, Dixon PC, Stebbins J, Zavatsky AB, and Theologis T. 2015. The relationship between quality of life and foot function in children with flexible flatfeet. Gait \& Posture 41:786-790.

Kothari A, Dixon PC, Stebbins J, Zavatsky AB, and Theologis T. 2016. Are flexible flat feet associated with proximal joint problems in children? Gait \& Posture 45:204-210.

Likert R. 1932. A technique for the measurement of attitudes. Archives of psychology 22:5-55.

Lin C-J, Lai K-A, Kuan T-S, and Chou Y-L. 2001. Correlating Factors and Clinical Significance of Flexible Flatfoot in Preschool Children. Journal of Pediatric Orthopaedics 21:378-382. 10.1097/00004694-200105000-00022

MacKenzie A, Rome K, and Evans AM. 2012. The efficacy of nonsurgical interventions for pediatric flexible flat foot: a critical review. Journal of Pediatric Orthopedics 32:830-834. http://dx.doi.org/10.1097/BPO.0b013e3182648c95

Mokkink LB, Terwee CB, Patrick DL, Alonso J, Stratford PW, Knol DL, Bouter LM, and De Vet HC. 2010. The COSMIN checklist for assessing the methodological quality of studies on measurement properties of health status measurement instruments: an international Delphi study. Quality of life research 19:539-549.

Okoli C, and Pawlowski SD. 2004. The Delphi method as a research tool: an example, design considerations and applications. Information \& management 42:15-29.

Pfeiffer M, Kotz R, Ledl T, Hauser G, and Sluga M. 2006. Prevalence of flat foot in preschool-aged children. Pediatrics 118:634-639.

Powell M, Seid M, and Szer IS. 2005. Efficacy of custom foot orthotics in improving pain and functional status in children with juvenile idiopathic arthritis: a randomized trial. The Journal of rheumatology 32:943-950.

Riccio I, Gimigliano F, Gimigliano R, Porpora G, and Iolascon G. 2009. Rehabilitative treatment in flexible flatfoot: a perspective cohort study. MUSCULOSKELETAL SURGERY 93:101. 10.1007/s12306-0090037-z

Rome K, Ashford RL, and Evans A. 2010. Non-surgical interventions for paediatric pes planus. Cochrane Database Systematic Review:CD006311. 10.1002/14651858.CD006311.pub2

Roth S, Roth A, Jotanovic Z, and Madarevic T. 2013. Navicular index for differentiation of flatfoot from normal foot. International orthopaedics 37:1107-1112.

Stavlas P, Grivas TB, Michas C, Vasiliadis E, and Polyzois V. 2005. The evolution of foot morphology in children between 6 and 17 years of age: a cross-sectional study based on footprints in a Mediterranean population. The Journal of Foot and Ankle Surgery 44:424-428.

Tudor A, Ruzic L, Sestan B, Sirola L, and Prpić T. 2009. Flat-footedness is not a disadvantage for athletic performance in children aged 11 to 15 years. Pediatrics 123:e386-e392.

Uden H, Scharfbillig R, and Causby R. 2017. The typically developing paediatric foot: how flat should it be? A systematic review. Journal of foot and ankle research 10:37. 10.1186/s13047-017-0218-1

Valmassy R, and Terrafranca N. 1986. The triplane wedge. An adjunctive treatment modality in pediatric biomechanics. Journal of the American Podiatric Medical Association 76:672-675.

Van der Linde H, Hofstad CJ, van Limbeek J, Postema K, and Geertzen JH. 2005. Use of the Delphi Technique for developing national clinical guidelines for prescription of lower-limb prostheses. Journal of rehabilitation research and development 42:693-704. 
529 Vernon W. 2009. The Delphi technique: A review. International Journal of Therapy \& Rehabilitation

530

531

532

533

534

535

536

537

538 16:69-76.

Vernon W, Parry A, and Potter M. 2003. Consensus obtained in a Delphi study of shoe wear pattern experiences amongst podiatrists. Journal of Forensic Identification 53:15-41.

Wenger DR, Mauldin D, Speck G, Morgan D, and Lieber RL. 1989. Corrective shoes and inserts as treatment for flexible flatfoot in infants and children. JBJS 71:800-810.

Whitford D, and Esterman A. 2007. A randomized controlled trial of two types of in-shoe orthoses in children with flexible excess pronation of the feet. Foot and Ankle International 28:715-723. WHO. 2016. International Classification of Diseases 10 (ICD-10). World Health Organisation 
Table $\mathbf{1}$ (on next page)

Participants' characteristics 


\begin{tabular}{lll}
\multirow{2}{*}{ Gender } & \multicolumn{1}{l}{8 Males } & $57.1 \%$ \\
\cline { 2 - 3 } & 6 Females & $42.8 \%$ \\
\hline Mean practice duration (mean + range) & 16.9 years & $8-28$ years \\
\hline Highest qualification & 7 PhD & $50 \%$ \\
\cline { 2 - 3 } & 2 Master's Degree & $0.14 \%$ \\
\cline { 2 - 3 } & 1 Graduate certificate & $0.07 \%$ \\
\cline { 2 - 3 } & 3 Bachelor's Degree & $0.21 \%$ \\
\hline Primary position & 6 clinicians & $42.8 \%$ \\
\cline { 2 - 3 } & 6 academics & $42.8 \%$ \\
\cline { 2 - 3 } & 2 researchers & $0.14 \%$ \\
\hline Secondary position & 5 clinicians & $0.36 \%$ \\
\cline { 2 - 3 } & 3 academics & $0.21 \%$ \\
\cline { 2 - 3 } & 2 researchers & $0.14 \%$ \\
\cline { 2 - 3 } & 4 No secondary position & $0.28 \%$ \\
\hline $\begin{array}{l}\text { Estimated average paediatric patient load } \\
\text { (mean }+ \text { range) }\end{array}$ & $57.9 \%$ & $20-100 \%$ \\
\hline $\begin{array}{l}\text { Estimated average paediatric consultations } \\
\text { per week (mean }+ \text { range) }\end{array}$ & 16 children \\
\hline $\begin{array}{l}\text { Estimated average number of orthosis } \\
\text { prescribed per week for children/ }\end{array}$ & $85.7 \%$ & $2-50$ children \\
\cline { 2 - 3 } \begin{tabular}{l} 
Adolescents \\
\cline { 2 - 3 }
\end{tabular} & $0.07 \%$ & $1-5$ pairs \\
\cline { 2 - 3 } & $0.07 \%$ & $11-15$ pairs \\
\hline
\end{tabular}

2 
Table 2 (on next page)

Statements reaching consensus ( $>70 \%$ ) in Round One 


\begin{tabular}{|c|c|c|}
\hline Category & Statement & $\begin{array}{l}\text { Level of } \\
\text { Consensus }\end{array}$ \\
\hline \multirow{2}{*}{$\begin{array}{l}\text { Determination of paediatric } \\
\text { flexible pes planus; }\end{array}$} & Visual/measured static foot posture assessment & $73.3 \%$ \\
\hline & $\begin{array}{l}\text { Foot posture tools (e.g. Foot posture index (FPI), } \\
\text { Paediatric flat foot proforma (pFFF)) }\end{array}$ & $80.0 \%$ \\
\hline Static foot posture measures; & $\begin{array}{l}\text { Rearfoot position (Resting Calcaneal Stance } \\
\text { Position-RCSP \& Neutral Calcaneal Stance } \\
\text { Position-NCSP) }\end{array}$ & $84.6 \%$ \\
\hline \multirow{3}{*}{$\begin{array}{l}\text { Foot function determination } \\
\text { in paediatric flexible pes } \\
\text { planus; }\end{array}$} & Visual gait analysis & $93.3 \%$ \\
\hline & Range of motion assessment & $100 \%$ \\
\hline & Muscle strength assessment & $93.3 \%$ \\
\hline \multirow{4}{*}{$\begin{array}{l}\text { Likeliness of FOs } \\
\text { prescription for paediatric } \\
\text { flexible pes planus; }\end{array}$} & $\begin{array}{l}\text { Severe abnormal foot posture (two Standard } \\
\text { Deviations from expected measure) }\end{array}$ & $78.6 \%$ \\
\hline & Activity limitation & $73.3 \%$ \\
\hline & Foot Pain & $93.3 \%$ \\
\hline & Lower limb pain & $73.3 \%$ \\
\hline $\begin{array}{l}\text { Weight/mass of the child } \\
\text { appropriate to initiate FOs } \\
\text { treatment for flexible pes } \\
\text { planus; }\end{array}$ & Weight does not influence the treatment decision & $92.3 \%$ \\
\hline \multirow{2}{*}{$\begin{array}{l}\text { Prescription variables used } \\
\text { for customised FOs for } \\
\text { flexible pes planus; }\end{array}$} & Neutral/vertical cast pour & $71.4 \%$ \\
\hline & Minimal arch fill & $76.9 \%$ \\
\hline \multirow{8}{*}{$\begin{array}{l}\text { Prescription variables NOT } \\
\text { to be used ( } 0 \% \text { use) for } \\
\text { customised FOs for flexible } \\
\text { pes planus; }\end{array}$} & Blake inverted device ( $>15$ degrees) & $84.6 \%$ \\
\hline & Everted cast pour & $91.6 \%$ \\
\hline & Blake inverted rearfoot post ( $>15$ degrees) & $90.0 \%$ \\
\hline & Everted rearfoot post & $90.0 \%$ \\
\hline & Rearfoot post with motion & $88.9 \%$ \\
\hline & Maximum arch fill & $72.7 \%$ \\
\hline & Inverted forefoot post & $70.0 \%$ \\
\hline & Everted forefoot post & $77.8 \%$ \\
\hline
\end{tabular}


Table 3(on next page)

Statements receiving agreement of $>70 \%$ from Round Two and Three of Delphi.

Abbreviations: WB: Wight bearing; WHO-ICD: World Health Organisation-International Classification of Diseases; STJ: Subtalar joint; MTJ: Midtarsal joint; and UCBL: University of California Biomechanics Laboratory. 
1 Table 3: Statements receiving agreement of $>\mathbf{7 0} \%$ from Round two and three of Delphi

Category

Flexible pes

planus

determination;

measures;

Foot function
determination in
paediatric flexible
pes planus;
The Balance tests
to assess foot
function;

Likeliness of FOs
prescription for
paediatric flexible
pes;

Static foot posture

Statement

Visual assessment of dynamic foot in gait

Dynamic WB and non-WB foot motion and/or measures

Foot Posture Index 6 (FPI-6)

Neurological assessments (Reflexes, sensation, tone and strength)

Single Limb Balance

Agreement

$85.7 \%$

$85.7 \%$

$100 \%$

$78.5 \%$

$71.4 \%$

Hopping ( $\mathrm{n}=$ dominant and non-dominant leg)

$78.5 \%$

Timed balance, standing on one leg (eyes open \& closed)

All balance tests for comprehensive assessment of functional impact rather than pes planus presence

Walk along straight line/marching/heel-toe gait (forwards and $\quad 78.5 \%$

backwards)

Running

Jumping

If dynamic foot function affected (instability in single leg stance, walking, running, turning, etc.)

In presence of symptoms (pain, reduced function, strength and $\quad 100 \%$ structure per WHO-ICD)

In presence of structural changes (hallux abducto valgus, hallux $\quad 71.4 \%$ limitus, etc.)

\begin{tabular}{ll}
\hline With foot posture related delayed milestones & $78.5 \%$ \\
\hline With parental concern, accompanied by affected function & $78.5 \%$ \\
\hline With gross pronation (apropulsive gait and low tone) & $100 \%$ \\
\hline With hereditary lower limb disorder/s changing function and & $92.8 \%$
\end{tabular} causing pain

If improvement in ICF (The International Classification of $\quad 71.4 \%$

Functioning, Disability and Health) outcomes

Symptoms (e.g. pain, general discomfort, reduced walking, poor $\quad 100 \%$ endurance and balance)

\begin{tabular}{ll}
\hline Plantar arch/fascia pain & $92.8 \%$ \\
\hline Heel pain & $78.5 \%$ \\
\hline Tibialis Posterior tendon pain & $100 \%$ \\
\hline Medial Tibial Stress Syndrome (MTSS) type symptoms & $100 \%$ \\
\hline Activity related pain & $92.8 \%$ \\
\hline $\begin{array}{l}\text { Other factors than age as extent/degree of deformity, type and } \\
\text { frequency of activity, and function }\end{array}$ & $92.8 \%$ \\
\hline Acquisition of motor skills rather than age & $71.4 \%$
\end{tabular}

Regarding child's

FOs use is

Acquisition of motor skills rather than age

$92.8 \%$

Presence of symptoms (foot and leg pain, affected function and

gross motor skill development)

FOs preferred, in:

Reduce symptoms

The aim of

prescribing FOs is to:

Reduce fatigue

$92.8 \%$

Improve gross motor skill

$85.7 \%$

Improve balance, stability, comfort, coordination, stamina and

$92.8 \%$ 


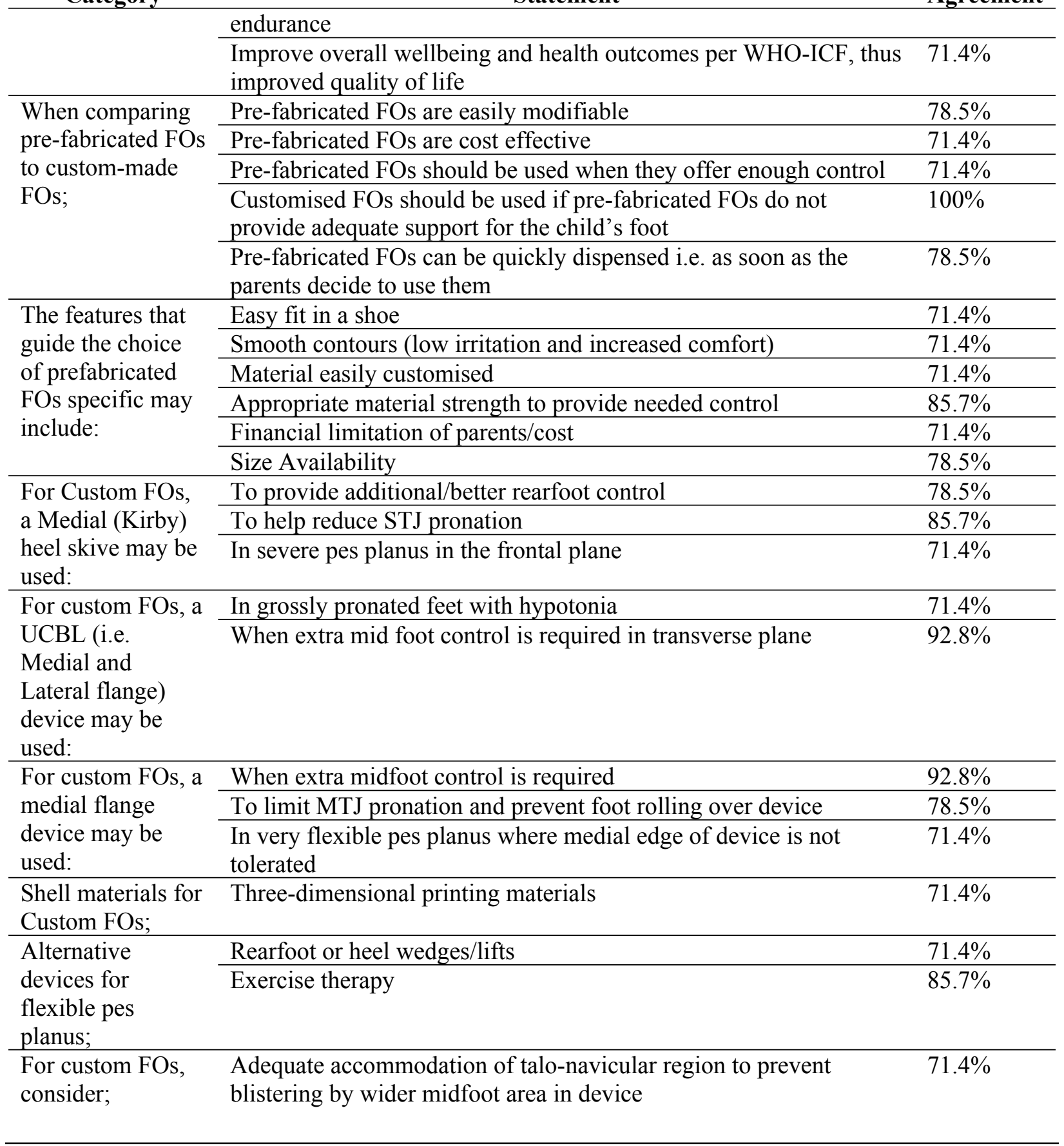

3 Abbreviations: WB: Wight bearing; WHO-ICD: World Health Organisation-International Classification of Diseases; STJ:

4 Subtalar joint; MTJ: Midtarsal joint; and UCBL: University of California Biomechanics Laboratory. 


\section{Figure 1}

Figure 1: Flow diagram of Delphi process with number of statements in each round

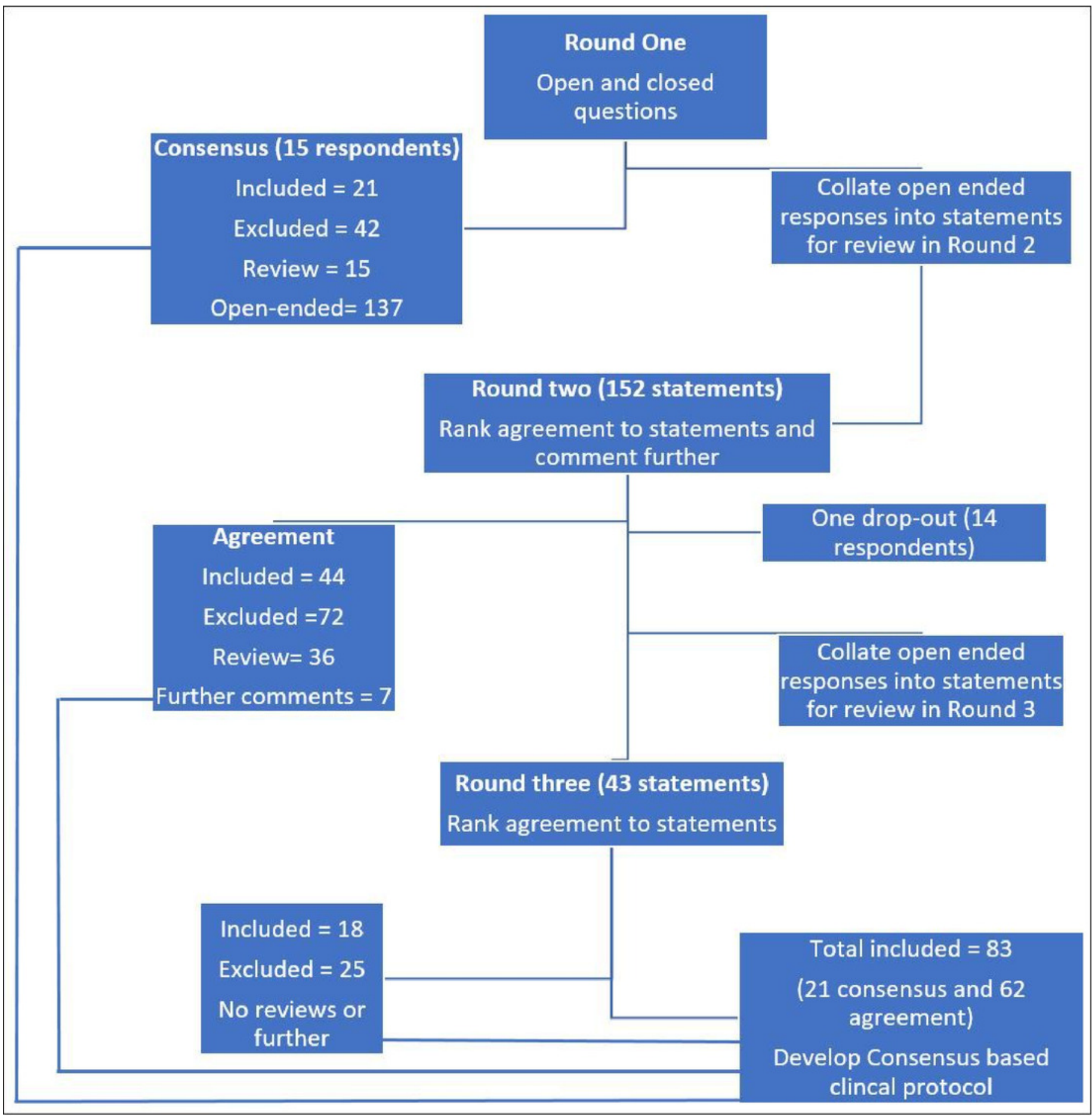




\section{Figure 2}

Figure 2: An example question from Round 2

3. The likeliness of intervening in paediatric flexible pes planus increases with:

Strongly Disagree

Disagree

Neutral

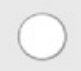

fascia $[n=2 / 15]$

Pain in heel $[n=2 / 15]$

Pain of Tibialis

Posterior tendon

$[n=1 / 15]$

Medial Tibial Stress

Syndrome (MTSS) like

symptoms $[n=1 / 15]$

Activity related pain

[ $n=1 / 15$ ]

Growing pains $[n=1 / 15]$

Night cramps $[n=1 / 15]$

Further Comments?
Agree Strongly Agree

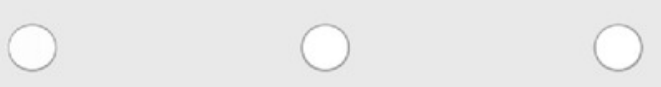

$\bigcirc$

$\bigcirc$

0

0
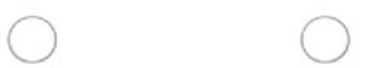

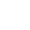

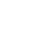




\section{Figure 3}

Figure 3: An example question from Round three

Statement in Round $\mathbf{2}$ - When prescribing Custom FOs for paediatric flexible pes planus, a Medial (Kirby) heel skive may be used:

\begin{tabular}{|l|l|l|}
\hline \multicolumn{1}{|c|}{ Statement } & Agreement \% & \multicolumn{1}{c|}{ Outcome } \\
\hline To provide additional/better rearfoot control & $84.6 \%$ & Accepted \\
\hline $\begin{array}{l}\text { To reduce pressure on midfoot making orthoses smaller and } \\
\text { easier to fit in footwear }\end{array}$ & $33.3 \%$ & Not accepted \\
\hline To help reduce STJ pronation & $69.2 \%$ & For review in Round 3 \\
\hline In severe pes planus in the frontal plane & $53.8 \%$ & For review in Round 3 \\
\hline
\end{tabular}

3 When prescribing Custom FOs for paediatric flexible pes planus, a Medial (Kirby) heel skive may be used:

\section{Strongly \\ Disagree}

To help reduce

STJ pronation

[69.2\% agreement]

In severe pes

planus in the

frontal plane

[53.8\% agreement]
Disagree

Neutral

Agree

Strongly Agree 
Figure 4

Figure 4: A protocol for paediatric flexible pes planus 


\section{Clinical protocol for paediatric flexible pes planus}

\section{Confirm Diagnosis}

Foot Posture Index - FPI-6 (Score + Posture $)=$ RCSP and NCSP =

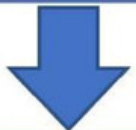

\section{Signs and Symptoms}

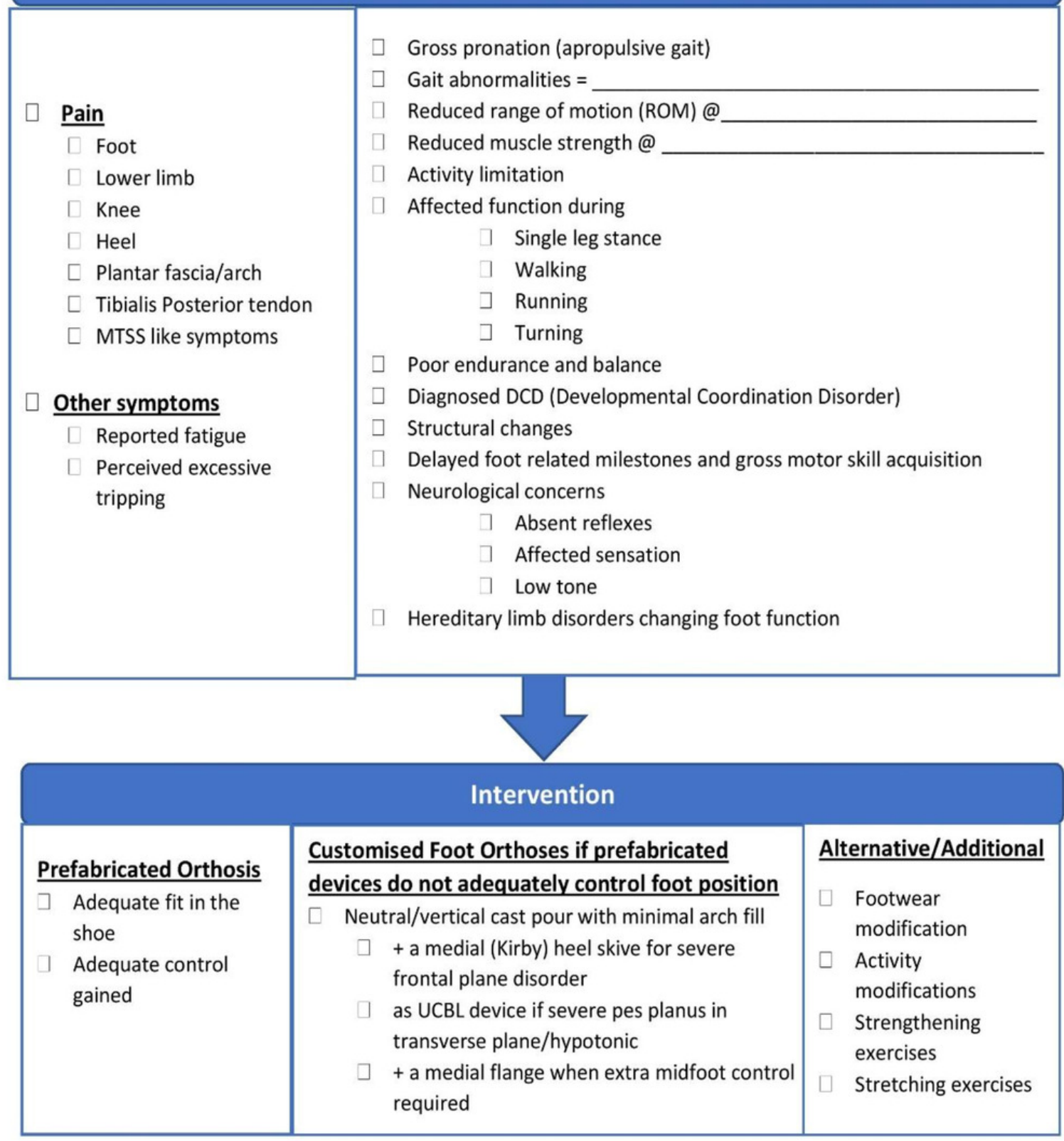

